\title{
STABILIZATION OF WALLS FOR NANO-WIRES OF FINITE LENGTH
}

\author{
Gilles Carbou ${ }^{1}$ and Stéphane LabBÉ ${ }^{2}$
}

\begin{abstract}
In this paper we study a one dimensional model of ferromagnetic nano-wires of finite length. First we justify the model by $\Gamma$-convergence arguments. Furthermore we prove the existence of wall profiles. These walls being unstable, we stabilize them by the mean of an applied magnetic field.
\end{abstract}

Mathematics Subject Classification. 35B35, 35K55.

Received March 30, 2009. Revised March 24, 2010 and September 27, 2010.

Published online December 2, 2010.

\section{INTRODUCTION}

This paper is concerned with stabilization of wall configurations in a mono-dimensional model of finite length nanowire. This kind of object can be found in nano electronic devices. The three dimensional model is the following (see $[2,11,20]$ ). We denote by $m: \mathbb{R}^{+} \times \Omega \longrightarrow \mathbb{R}^{3}$ the magnetic moment, defined on the ferromagnetic domain $\Omega$. We assume that the material is saturated, so that $m$ satisfies the constraint:

$$
\text { for almost every }(t, X) \in \mathbb{R}^{+} \times \Omega,|m(t, X)|=1 \text {. }
$$

The magnetic moment links the magnetic induction $B$ with the magnetic field $H$ by the relation $B=H+\bar{m}$, where $\bar{m}$ is the extension of $m$ by zero outside $\Omega$. The behavior of $m$ is governed by the Landau-Lifschitz equation:

$$
\left\{\begin{array}{l}
\frac{\partial m}{\partial t}=-m \wedge H_{e}-m \wedge\left(m \wedge H_{e}\right), \\
H_{e}=A^{2} \Delta m+H_{d}(m)+H_{a} \\
\frac{\partial m}{\partial \mathbf{n}}=0 \text { on } \partial \Omega
\end{array}\right.
$$

where

- $A^{2}$ is the exchange constant, depending on the material;

Keywords and phrases. Landau-Lifschitz equation, control, stabilization.

1 MAB, UMR 5466, CNRS, Université Bordeaux 1, 351 cours de la Libération, 33405 Talence Cedex, France.

carbou@math.u-bordeaux1.fr

2 Université Joseph Fourier, Laboratoire Jean Kuntzmann, CNRS, UMR 5224, 51 rue des Mathématiques, B.P. 53, 38041

Grenoble Cedex 9, France. stephane.labbe@imag.fr 
- the demagnetizing field $H_{d}(m)$ is characterized by

$$
\operatorname{curl} H_{d}(m)=0 \text { and } \operatorname{div}\left(H_{d}(m)+\bar{m}\right)=0 \text { in } \mathbb{R}^{3} .
$$

We remark that $-H_{d}(m)$ is the orthogonal projection of $\bar{m}$ (for the $L^{2}$ inner product) onto the curl free vector fields, so that

$$
\left\|H_{d}(m)\right\|_{L^{2}\left(\mathbb{R}^{3}\right)} \leq\|m\|_{L^{2}(\Omega)}
$$

- $H_{a}$ is an applied magnetic field;

- $\mathbf{n}$ is the outward unit normal on $\partial \Omega$.

The effective field is derived from the micromagnetism energy: $H_{e}=-\partial_{m} \mathcal{E}_{\text {mic }}$ with

$$
\mathcal{E}_{\text {mic }}(m)=\frac{A^{2}}{2} \int_{\Omega}|\nabla m|^{2} \mathrm{~d} X+\frac{1}{2} \int_{\mathbb{R}^{3}}\left|H_{d}(m)\right|^{2} \mathrm{~d} X-\int_{\Omega} H_{a} \cdot m \mathrm{~d} X .
$$

Existence of weak solutions for (1.1) is established in [5,14,19]. Existence of strong solutions is proved in $[6,7]$. Numerical simulations are performed in [15]. For thin domains, equivalent 2-d models are justified in $[1,4,10,16]$. For nanowires, 1 -d models are discussed in $[8,9,13,17]$.

In this paper we deal with ferromagnetic nanowires. We assume that the wire is a cylinder of length $2 L$ and radius $\eta$. Taking the limit by $\Gamma$-convergence arguments when $\eta$ tends to zero, we obtain an asymptotic one dimensional model (see Sect. 2).

After renormalization, the one dimensional wire is assimilated to the segment $]-\frac{L}{A \sqrt{2}}, \frac{L}{A \sqrt{2}}\left[e_{1}\right.$, where $\left(e_{1}, e_{2}, e_{3}\right)$ is the canonical basis of $\mathbb{R}^{3}$. The magnetic moment $m$ is then defined on $\left.\mathbb{R}_{t}^{+} \times\right]-\frac{L}{A \sqrt{2}}, \frac{L}{A \sqrt{2}}[$. The equivalent demagnetizing field (after renormalization) is given by

$$
h_{d}(m)=-m_{2} e_{2}-m_{3} e_{3},
$$

i.e. $-h_{d}$ is the orthogonal projection onto the plane orthogonal to the wire. In addition we assume that we apply a magnetic field in the direction of the wire axis, and we denote by $h_{a}$ its renormalized intensity. Therefore we deal with the following system:

$$
\left\{\begin{array}{l}
\frac{\partial m}{\partial t}=-m \wedge H_{e}-m \wedge\left(m \wedge H_{e}\right), \\
H_{e}=\frac{\partial^{2} m}{\partial x^{2}}-m_{2} e_{2}-m_{3} e_{3}+h_{a} e_{1}, \\
\frac{\partial m}{\partial x}\left(t,-\frac{L}{A \sqrt{2}}\right)=\frac{\partial m}{\partial x}\left(t, \frac{L}{A \sqrt{2}}\right)=0, \\
\text { with } \left.m: \mathbb{R}_{t}^{+} \times\right]-\frac{L}{A \sqrt{2}}, \frac{L}{A \sqrt{2}}\left[\longrightarrow \mathbb{R}^{3},|m|=1,\right.
\end{array}\right.
$$

associated to the energy

$$
\mathcal{E}(m)=\int_{-L / A \sqrt{2}}^{L / A \sqrt{2}}\left(\frac{1}{2}\left|\partial_{x} m\right|^{2}+\frac{1}{2}\left(\left|m_{2}\right|^{2}+\left|m_{3}\right|^{2}\right)-h_{a} m_{1}\right) \mathrm{d} x .
$$


In $[8,9]$, we studied a similar model of infinite nanowire. After renormalization, the model was the following: the wire is assimilated to the real axis $\mathbb{R} e_{1}$, and the one dimensional Landau-Lifschitz equation is in this case:

$$
\left\{\begin{array}{l}
\frac{\partial m}{\partial t}=-m \wedge H_{e}-m \wedge\left(m \wedge H_{e}\right) \\
H_{e}=\frac{\partial^{2} m}{\partial x^{2}}-m_{2} e_{2}-m_{3} e_{3}+h_{a} e_{1} \\
\text { with } m: \mathbb{R}_{t}^{+} \times \mathbb{R}_{x} \longrightarrow \mathbb{R}^{3},|m|=1
\end{array}\right.
$$

This system is invariant by translation in the $x$ variable and by rotation around the wire axis, that is, if $m$ is a solution of (1.4), then for $\sigma$ and $\tau$ in $\mathbb{R},(t, x) \mapsto \rho_{\tau}(m(t, x-\sigma))$ is another solution of (1.4), where $\rho_{\tau}$ is the rotation of angle $\tau$ around the $e_{1}$ axis:

$$
\rho_{\tau}=\left(\begin{array}{ccc}
1 & 0 & 0 \\
0 & \cos \tau & -\sin \tau \\
0 & \sin \tau & \cos \tau
\end{array}\right) .
$$

We dealt with wall configurations for this model, that is solutions separating a left hand side domain where the magnetization is closed to $-e_{1}$ to a right hand side domain where the magnetization is closed to $+e_{1}$. Such solutions are described by rotations and translations of the canonical profile $x \mapsto(\operatorname{th} x, 1 / \operatorname{ch} x, 0)$. In $[8,9]$, we proved the asymptotic stability and the controllability for these configurations.

Here, in the case of a finite wire, the situation is quite different.

Definition 1.1. We call canonical wall profile a static solution for $(1.3)$ of the form $M_{0}=\left(\sin \theta_{0}, \cos \theta_{0}, 0\right)$ such that $\theta_{0}:\left[-\frac{L}{A \sqrt{2}}, \frac{L}{A \sqrt{2}}\right] \longrightarrow[-\pi / 2, \pi / 2]$ is a non decreasing map satisfying $\theta_{0}\left(-\frac{L}{A \sqrt{2}}\right)<0<\theta_{0}\left(\frac{L}{A \sqrt{2}}\right)$.

In the following theorem, we claim that the wall profiles exist if and only if the wire is long enough compared to the exchange length:

Theorem 1.2. There exists a canonical wall profile if and only if $\frac{L}{A \sqrt{2}}>\pi / 2$. This profile is unique and is centered in the middle of the wire, that is $\theta_{0}(0)=0$. In addition, $\theta_{0}$ is odd.

Remark 1.1. In the infinite wire case, the corresponding canonical profiles are obtained taking $\theta_{0}(x)=$ Arcsinth $x$, and all its translation in the $x$ variable (because of the invariance of (1.4) by translation). In the finite wire case, we only have invariance by rotation, and we loose the invariance by translation, so we have only one canonical profile.

Concerning the stability, we obtained in [8] the stability of the wall profile. In that case, the invariance by rotation-translation induces that 0 is a double eigenvalue of the linearized version of (1.4) around the wall profile. In the finite wire case, the lack of invariance by translation induces that the linearized version of (1.3) around the canonical profile has one negative eigenvalue, and therefore we can prove that the wall profile given by Theorem 1.2 is unstable.

Theorem 1.3. Assume that $\frac{L}{A \sqrt{2}}>\pi / 2$. Let $\theta_{0}$ given by Theorem 1.2 . The static solution $M_{0}=\left(\sin \theta_{0}, \cos \theta_{0}, 0\right)$ in linearly unstable for the Landau-Lifschitz equation (1.3) with $h_{a}=0$.

Remark 1.2. This phenomenon was expected. Let us consider a small translation of the centered wall. Without energetic cost, the Landau-Lifschitz equation induces then a displacement of the wall and pushes it outside the wire. Then the magnetic moment tends to $+\pi / 2$ or $-\pi / 2$ (i.e. the minimizers of the ferromagnetism energy $\mathcal{E}$ ). In the case of an infinite wire, obviously, this translation cannot make the wall disappear, and we have stability.

We prove now a stabilization result. We control the system by an applied field parallel to the wire axis. 


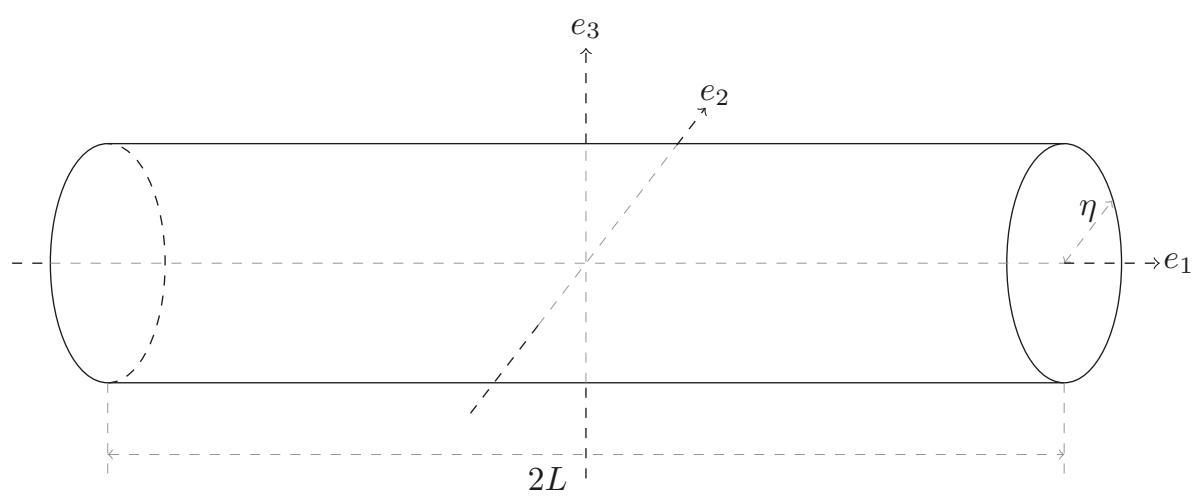

Figure 1. Domain $\Omega_{\eta}$.

Theorem 1.4. Let $L$ and $A$ as in Theorem 1.2 , and let $M_{0}=\left(\sin \theta_{0}, \cos \theta_{0}, 0\right)$ be the canonical profile given by this theorem. We consider the following control:

$$
h(m(t, .))=\left[-\frac{A}{\sqrt{2} L} \int_{-\frac{L}{A \sqrt{2}}}^{\frac{L}{A \sqrt{2}}} m_{1}(t, s) \mathrm{d} s\right] .
$$

Then $M_{0}$ is stable for the Landau Lifschitz equation controlled with the applied field $h_{a}=h(m)$.

Remark 1.3. The control given here is quite natural: for example when the wall is translated to the right hand side, then the average of the profile first component is negative and our applied field $h e_{1}$ (with $h>0$ ) pushes the wall to the left hand side.

The paper is organized as follows. In Section 2 we justify the one dimensional model by $\Gamma$-convergence arguments. In Section 3 we prove the existence of a canonical wall profile. We address the unstability of this profile in Section 4 by linearization of the Landau-Lifschitz equation. The last section is devoted to the stabilization of the wall by a convenient applied magnetic field.

\section{MODELIZATION}

In this section, we address the justification of the one dimensional model by $\Gamma$ convergence arguments.

At the beginning we deal with the three-dimensional static case. The finite $3 \mathrm{~d}$ wire is the cylinder $\Omega_{\eta}=$ ] $-L, L\left[\times B_{2}(0, \eta)\right.$, where $B_{d}(X, r)$ is the ball of radius $r$ and center $X$ in $\mathbb{R}^{d}$. We assume that we apply on the wire a magnetic field in the direction $e_{1}: H_{a}=h_{a} e_{1}$, where $h_{a} \in \mathbb{R}$. See Figure 1 .

The micromagnetism energy on $\Omega_{\eta}$ is given by

$$
\mathcal{E}_{\eta}(v)=\frac{A^{2}}{2} \int_{\Omega_{\eta}}|\nabla v|^{2} \mathrm{~d} X+\frac{1}{2} \int_{\mathbb{R}^{3}}\left|H_{d}(v)\right|^{2} \mathrm{~d} X-h_{a} \int_{\Omega_{\eta}} v \cdot e_{1} \mathrm{~d} X \text { for } v \in H^{1}\left(\Omega_{\eta} ; S^{2}\right) .
$$

The static configurations satisfy the minimization problem:

$$
\left\{\begin{array}{l}
\text { find } u \text { in } H^{1}\left(\Omega_{\eta} ; S^{2}\right) \text { such that } \\
\mathcal{E}_{\eta}(u)=\min _{v \in H^{1}\left(\Omega_{\eta} ; S^{2}\right)} \mathcal{E}_{\eta}(v)
\end{array}\right.
$$


In order to work with a fixed domain, we introduce the following rescaling: for $v \in H^{1}\left(\Omega_{\eta} ; S^{2}\right)$, we define $\underline{v} \in H^{1}\left(\Omega_{1} ; S^{2}\right)$ by

$$
\underline{v}(x, y, z)=v(x, \eta y, \eta z) .
$$

In addition, for $\underline{v} \in H^{1}\left(\Omega_{1} ; S^{2}\right)$, we denote by $\underline{H_{d}^{\eta}}(\underline{v})$ the rescaled demagnetizing field:

$$
\underline{H_{d}^{\eta}}(\underline{v})(x, y, z)=H_{d}(v)(x, \eta y, \eta z),
$$

where $v \in H^{1}\left(\Omega_{\eta} ; S^{2}\right)$ is deduced from $\underline{v}$ by $v(x, y, z)=\underline{v}(x, y / \eta, z / \eta)$.

We deal with the rescaled energy defined for $\underline{v} \in H^{1}\left(\Omega_{1} ; S^{2}\right)$ by $\underline{\mathcal{E}}_{\eta}(\underline{v})=\frac{1}{\eta^{2}} \mathcal{E}_{\eta}(v)$ :

$$
\underline{\mathcal{E}}_{\eta}(\underline{v})=\frac{A^{2}}{2} \int_{\Omega_{1}}\left(\left|\partial_{x} \underline{v}\right|^{2}+\frac{1}{\eta^{2}}\left|\partial_{y} \underline{v}\right|^{2}+\frac{1}{\eta^{2}}\left|\partial_{z} \underline{v}\right|^{2}\right) \mathrm{d} X+\left.\frac{1}{2} \int_{\mathbb{R}^{3}} \underline{\mid H_{d}^{\eta}} \underline{v)}\right|^{2} \mathrm{~d} X-h_{a} \int_{\Omega_{1}} \underline{v} \cdot e_{1} \mathrm{~d} X
$$

and the problem (2.1) is rescaled in the following equivalent problem:

$$
\left\{\begin{array}{l}
\text { find } \underline{u} \text { in } H^{1}\left(\Omega_{1} ; S^{2}\right) \text { such that } \\
\underline{\mathcal{E}}_{\eta}(\underline{u})=\min _{\underline{v} \in H^{1}\left(\Omega_{1} ; S^{2}\right)} \underline{\mathcal{E}}_{\eta}(\underline{v})
\end{array}\right.
$$

Proposition 2.1. For all $\left(\underline{v}_{\eta}\right)_{\eta \in \mathbb{R}_{*}^{+}}$in $H^{1}\left(\Omega_{1} ; S^{2}\right)$, sequence of minimizers of $(2.2),\left(\underline{\mathcal{E}}_{\eta}\left(\underline{v}_{\eta}\right)\right)_{\eta \in \mathbb{R}_{*}^{+}}$is a bounded sequence of $\mathbb{R}$.

Proof. Let $\left(\underline{v}_{\eta}\right)_{\eta \in \mathbb{R}_{*}^{+}}$be a sequence of minimizers of $(2.2)$ in $H^{1}\left(\Omega_{1} ; S^{2}\right)$. We denote by $v_{\eta}$ the rescaled of $\underline{v}_{\eta}$ : $v_{\eta}(x, y, z)=\underline{v}_{\eta}(x, y / \eta, z / \eta)$.

In order to exhibit an upper bound for $\underline{\mathcal{E}}_{\eta}\left(\underline{v}_{\eta}\right)$, we write:

$$
\forall \eta \in \mathbb{R}_{*}^{+}, \mathcal{E}_{\eta}\left(v_{\eta}\right) \leq \mathcal{E}_{\eta}\left(e_{1}\right)
$$

then

$$
\begin{aligned}
\mathcal{E}_{\eta}\left(v_{\eta}\right) & \leq-2 h_{a} \eta^{2} \pi L+\frac{1}{2} \int_{\mathbb{R}^{3}}\left|H_{d}\left(e_{1}\right)\right|^{2} \mathrm{~d} X \\
& \leq 2 \eta^{2} L \pi\left(1-h_{a}\right) .
\end{aligned}
$$

Then, the lower bound is obtained by canceling the positive contributions of the energy and maximizing the external contribution:

So, we can conclude

$$
-\eta^{2} 2 h_{a} \pi L \leq \mathcal{E}_{\eta}\left(v_{\eta}\right) .
$$

$$
-2 h_{a} \pi L \leq \underline{\mathcal{E}}_{\eta}\left(\underline{v}_{\eta}\right) \leq 2 L \pi\left(1-h_{a}\right) .
$$

We introduce the limit energy: we define $\underline{H}^{1}\left(\Omega_{1} ; S^{2}\right)$ by

$$
\underline{H}^{1}\left(\Omega_{1} ; S^{2}\right)=\left\{u \in H^{1}\left(\Omega_{1} ; S^{2}\right), \partial_{y} u=\partial_{z} u=0\right\} .
$$

For $\underline{v} \in \underline{H}^{1}\left(\Omega_{1} ; S^{2}\right)$, we set:

$$
\begin{aligned}
\underline{\mathcal{E}}(\underline{v}) & =\frac{A^{2}}{2} \int_{\Omega_{1}}\left|\partial_{x} \underline{v}\right|^{2} \mathrm{~d} X+\frac{1}{4} \int_{\Omega_{1}}\left(\left|\underline{v}_{2}\right|^{2}+\left|\underline{v}_{3}\right|^{2}\right) \mathrm{d} X-h_{a} \int_{\Omega_{1}} \underline{v} \cdot e_{1} \mathrm{~d} X \\
& =\frac{A^{2} \pi}{2} \int_{]-L, L[}\left|\partial_{x} \underline{v}\right|^{2} \mathrm{~d} x+\frac{\pi}{4} \int_{]-L, L[}\left(\left|\underline{v}_{2}\right|^{2}+\left|\underline{v}_{3}\right|^{2}\right) \mathrm{d} x-\pi h_{a} \int_{]-L, L[} \underline{v} \cdot e_{1} \mathrm{~d} x .
\end{aligned}
$$


The limit minimization problem is given by:

$$
\left\{\begin{array}{l}
\text { find } u \text { in } \underline{H}^{1}\left(\Omega_{1}, S^{2}\right) \text { such that } \\
\underline{\mathcal{E}}(u)=\min _{v \in \underline{H}^{1}\left(\Omega_{1}, S^{2}\right)} \underline{\mathcal{E}}(v) .
\end{array}\right.
$$

The main result of this section is the following:

Theorem 2.1. $\underline{\mathcal{E}}_{\eta} \Gamma$-converges to $\underline{\mathcal{E}}$ in sense of $H^{1}\left(\Omega_{1} ; \mathbb{R}^{3}\right)$ weak, it is to say:

(i) (lower semi continuity) for all sequence $\left(\underline{v}_{\eta}\right)_{\eta \in \mathbb{R}_{*}^{+}}$of $H^{1}\left(\Omega_{1} ; S^{2}\right)$ such that $\underline{v}_{\eta} \rightarrow \underline{v}_{0}$ in $H^{1}\left(\Omega_{1} ; \mathbb{R}^{3}\right)$ weakly when $\eta \rightarrow 0$, and $\left(\underline{\mathcal{E}}_{\eta}\left(\underline{v}_{\eta}\right)\right)_{\eta \in \mathbb{R}_{*}^{+}}$is bounded, then the limit $\underline{v}_{0}$ is an element of $\underline{H}^{1}\left(\Omega_{1} ; S^{2}\right)$ such that:

$$
\liminf _{\eta \rightarrow 0} \underline{\mathcal{E}}_{\eta}\left(\underline{v}_{\eta}\right) \geq \underline{\mathcal{E}}\left(\underline{v}_{0}\right)
$$

(ii) (reconstruction) for all $\underline{u}_{0} \in \underline{H}^{1}\left(\Omega_{1} ; S^{2}\right)$, there exists $\left(\underline{v}_{\eta}\right)_{\eta \in \mathbb{R}_{*}^{+}}$, sequence of $H^{1}\left(\Omega_{1} ; S^{2}\right)$, such that:

$$
\lim _{\eta \rightarrow 0} \underline{v}_{\eta}=\underline{u}_{0}
$$

and

$$
\limsup _{\eta \rightarrow 0} \underline{\mathcal{E}}_{\eta}\left(\underline{v}_{\eta}\right) \leq \underline{\mathcal{E}}\left(\underline{u}_{0}\right)
$$

Proof. (i) Lower semi-continuity. Let $\left(\underline{v}_{\eta}\right)_{\eta}$ be a sequence in $H^{1}\left(\Omega_{1} ; \mathcal{S}^{2}\right)$ such that $\underline{v}_{\eta}$ tends to $\underline{v}$ in $H^{1}\left(\Omega_{1}\right)$ weak and such that there exists $c$ such that for all $\eta$

$$
\underline{\mathcal{E}}_{\eta}\left(\underline{v}_{\eta}\right) \leq c
$$

Extracting a subsequence, we can assume that $\underline{v}_{\eta}$ tends to $\underline{v}$ almost everywhere, so $|\underline{v}|=1$ a.e.

Using (2.4), we remark that since $\left|\int_{\Omega_{1}} \underline{v}_{\eta} e_{1}\right| \leq \operatorname{meas}\left(\Omega_{1}\right)$,

$$
\left\|\partial_{y} \underline{v}_{\eta}\right\|_{L^{2}\left(\Omega_{1}\right)}^{2}+\left\|\partial_{z} \underline{v}_{\eta}\right\|_{L^{2}\left(\Omega_{1}\right)}^{2} \leq C \eta^{2},
$$

so $\partial_{y} \underline{v}=\partial_{z} \underline{v}=0$, that is

$$
\underline{v} \in \underline{H}^{1}\left(\Omega_{1} ; S^{2}\right) .
$$

From straightforward arguments,

$$
\begin{aligned}
& \frac{A^{2}}{2} \int_{\Omega_{1}}\left|\partial_{x} \underline{v}\right|^{2} \mathrm{~d} X-h_{a} \int_{\Omega_{1}} \underline{v} \cdot e_{1} \mathrm{~d} X \\
& \quad \leq \lim \inf \left(\frac{A^{2}}{2} \int_{\Omega_{1}}\left(\left|\partial_{x} \underline{v}_{\eta}\right|^{2}+\frac{1}{\eta^{2}}\left|\partial_{y} \underline{v}_{\eta}\right|^{2}+\frac{1}{\eta^{2}}\left|\partial_{z} \underline{v}_{\eta}\right|^{2}\right) \mathrm{d} X-h_{a} \int_{\Omega_{1}} \underline{v}_{\eta} \cdot e_{1} \mathrm{~d} X\right) .
\end{aligned}
$$


Concerning the demagnetizing field, the sequence $\left(\underline{H}_{d}^{\eta}\left(\underline{v}_{\eta}\right)\right)_{\eta}$ is bounded in $L^{2}\left(\mathbb{R}^{3}\right)$. Indeed, we denote by $v_{\eta}$ the rescaled of $\underline{v}_{\eta}: v_{\eta}(x, y, z)=\underline{v}_{\eta}(x, y / \eta, z / \eta)$. We have

$$
\begin{aligned}
\left\|\underline{H} \underline{H}_{d}^{\eta}\left(\underline{v}_{\eta}\right)\right\|_{L^{2}\left(\mathbb{R}^{3}\right)} & =\frac{1}{\eta}\left\|H_{d}\left(v_{\eta}\right)\right\|_{L^{2}\left(\mathbb{R}^{3}\right)} \text { (by change of variable in the integral) } \\
& \leq \frac{1}{\eta}\left\|v_{\eta}\right\|_{L^{2}\left(\mathbb{R}^{3}\right)}\left(\text { since }-H_{d} \text { is an orthogonal projection in } L^{2}\right) \\
& \leq \frac{1}{\eta}\left(2 L \pi \eta^{2}\right)^{\frac{1}{2}}\left(\text { since }\left|v_{\eta}\right|=1\right) \\
& \leq \sqrt{2 \pi L} .
\end{aligned}
$$

So, up to the extraction of a subsequence, we can assume that $\underline{H}_{d}^{\eta}\left(\underline{v}_{\eta}\right) \rightarrow \underline{H}$ for the weak topology in $L^{2}\left(\mathbb{R}^{3}\right)$.

We denote the coordinates of $\underline{H}_{d}^{\eta}\left(\underline{v}_{\eta}\right)$ and those of $\underline{H}$ by:

$$
\left.\underline{H_{d}^{\eta}} \underline{v}_{\eta}\right)=\left(\begin{array}{c}
h_{\eta}^{1} \\
h_{\eta}^{2} \\
h_{\eta}^{3}
\end{array}\right) \text { and } \underline{H}=\left(\begin{array}{c}
h^{1} \\
h^{2} \\
h^{3}
\end{array}\right)
$$

Writing (1.2) in the rescaled coordinates, we obtain first that

$$
\eta \partial_{x}\left(h_{\eta}^{1}+\underline{v}_{\eta}^{1}\right)+\partial_{y}\left(h_{\eta}^{2}+\underline{v}_{\eta}^{2}\right)+\partial_{z}\left(h_{\eta}^{3}+\underline{v}_{\eta}^{3}\right)=0
$$

so taking the weak limit, we have:

$$
\partial_{y}\left(h^{2}+\underline{\underline{v}}^{2}\right)+\partial_{z}\left(h^{3}+\underline{v}^{3}\right)=0 .
$$

In addition, from the curl free condition, we obtain that

$$
\left\{\begin{array}{l}
\partial_{y} h_{\eta}^{3}-\partial_{z} h_{\eta}^{2}=0 \\
\eta \partial_{x} h_{\eta}^{2}-\partial_{y} h_{\eta}^{1}=0 \\
\eta \partial_{x} h_{\eta}^{3}-\partial_{z} h_{\eta}^{1}=0
\end{array}\right.
$$

so, taking the weak limit we get

$$
\partial_{y} h^{1}=\partial_{z} h^{1}=0 \text { so } h^{1}=0, \text { since } h^{1} \in L^{2}\left(\mathbb{R}^{3}\right),
$$

and

So $\underline{H}$ is deduced from $\underline{v}$ by the relations:

$$
\partial_{y} h^{3}-\partial_{z} h^{2}=0
$$

$$
\left\{\begin{array}{l}
\partial_{y}\left(h^{2}+\underline{v}^{2}\right)+\partial_{z}\left(h^{3}+\underline{v}^{3}\right)=0 \\
\partial_{y} h^{3}-\partial_{z} h^{2}=0 \\
h^{1}=0
\end{array}\right.
$$


that is for a fixed $x, \underline{H}(x, \cdot)$ is defined from $\underline{v}(x, \cdot)$ by the 2 -d demagnetizing field. In the following lemma we calculate the 2-d demagnetizing field induced by a constantly magnetized 2-d disk.

Lemma 2.2. Let $\xi=\left(\xi_{2}, \xi_{3}\right) \in \mathbb{R}^{2}$ be a fixed vector. Let $V=\left(V^{2}, V^{3}\right) \in L^{2}\left(\mathbb{R}^{2} ; \mathbb{R}^{2}\right)$ such that

$$
\left\{\begin{array}{l}
\operatorname{curl}_{2 D} V:=\partial_{y} V^{3}-\partial_{z} V^{2}=0 \\
\operatorname{div}{ }_{2 D}(V+\bar{\xi}):=\partial_{y}\left(V^{2}+\overline{\xi_{2}}\right)+\partial_{z}\left(V^{3}+\overline{\xi_{3}}\right)=0
\end{array}\right.
$$

where $\bar{\xi}(y, z)=\xi \chi_{B(0,1)}(y, z)$ (we denote by $\chi_{B(0,1)}$ the characteristic function of $\left.B(0,1)\right)$. Then

$$
V(y, z)=\left\{\begin{array}{l}
-\frac{1}{2} \xi \text { if }(y, z) \in B_{2}(0,1), \\
\frac{1}{2} \frac{1}{\left(y^{2}+z^{2}\right)^{2}}\left(\begin{array}{c}
\xi_{2}\left(y^{2}-z^{2}\right)+2 \xi_{3} y z \\
-\xi_{3}\left(y^{2}-z^{2}\right)-2 \xi_{2} y z
\end{array}\right) \text { if }(y, z) \notin B_{2}(0,1),
\end{array}\right.
$$

and

$$
\int_{\mathbb{R}^{2}}|V|^{2} \mathrm{~d} y \mathrm{~d} z=\frac{\pi}{2}|\xi|^{2} .
$$

Proof. We have (see [3] or [14]), for $X=(y, z) \in \mathbb{R}^{2}$ :

$$
V(X)=\frac{1}{2 \pi} \int_{Y \in \partial B_{2}(0,1)} \frac{X-Y}{|X-Y|^{2}} \xi \cdot \nu(Y) \mathrm{d} \sigma(Y),
$$

where $\nu$ is the unit normal on $\partial B_{2}(0,1)$.

Using complex notations, we write $V(X)=V^{2}(X)+\mathrm{i} V^{3}(X), X=y+\mathrm{i} z, \xi=\xi_{2}+\mathrm{i} \xi_{3}$ and we have:

$$
V(X)=\frac{1}{2 \mathrm{i} \pi} \int_{z \in \mathcal{C}(0,1)} \frac{\xi+w^{2} \bar{\xi}}{2 \bar{X} w\left(w-\frac{1}{\bar{X}}\right)} \mathrm{d} w .
$$

If $|X|<1$, the only pole is 0 , and by the residue formula, $V(X)=-\frac{1}{2} \xi$. If $|X|>1$, there are two poles, 0 and $1 / \bar{X}$, and we obtain $V(X)=\frac{1}{2} \frac{\bar{\xi}}{X^{2}}$ which is equivalent to $(2.6)$.

In addition, by a direct calculation, we obtain that:

$$
\int_{\mathbb{R}^{2}}|V(y, z)|^{2} \mathrm{~d} y \mathrm{~d} z=\frac{\pi}{2}|\xi|^{2} .
$$

We apply the previous lemma to calculate the weak limit $\underline{H}$ :

$$
\underline{H}(x, y, z)=\left\{\begin{array}{l}
-\frac{1}{2}\left(\begin{array}{c}
0 \\
\underline{v}^{2}(x) \\
\underline{v}^{3}(x)
\end{array}\right) \text { if }(y, z) \in B_{2}(0,1), \\
\frac{1}{2} \frac{1}{\left(y^{2}+z^{2}\right)^{2}}\left(\begin{array}{c}
0 \\
\underline{v}^{2}(x)\left(y^{2}-z^{2}\right)-2 \underline{v}^{3}(x) y z \\
-\underline{v}^{3}\left(y^{2}-z^{2}\right)-2 \underline{v}^{2} y z
\end{array}\right) \text { if }(y, z) \notin B_{2}(0,1),
\end{array}\right.
$$

and by a direct calculation,

$$
\int_{\mathbb{R}^{3}}|\underline{H}(x, y, z)|^{2} \mathrm{~d} x \mathrm{~d} y \mathrm{~d} z=\frac{\pi}{2} \int_{\mathbb{R}}\left(\left|\underline{v}^{2}(x)\right|^{2}+\left|\underline{v}^{3}(x)\right|^{2}\right) \mathrm{d} x .
$$


We remark now that since $-H_{d}$ is an orthogonal projection for the $L^{2}$ inner product,

$$
\int_{\mathbb{R}^{3}}\left|\underline{H}_{d}^{\eta}\left(\underline{v}_{\eta}\right)\right|^{2} \mathrm{~d} X=-\int_{\Omega_{1}} \underline{H_{d}^{\eta}}\left(\underline{v}_{\eta}\right) \cdot \underline{v}_{\eta} \mathrm{d} X .
$$

Thus we can take the limit in this integral, and we obtain that:

$$
\int_{\mathbb{R}^{3}}\left|\underline{H}_{d}^{\eta}\left(\underline{v}_{\eta}\right)\right|^{2} \mathrm{~d} X \longrightarrow-\int_{\Omega_{1}} \underline{H} \cdot \underline{v} \mathrm{~d} X=\left.\int_{\mathbb{R}^{3}} \underline{\mid H}\right|^{2} \mathrm{~d} X .
$$

So, in fact, $\underline{H_{d}^{\eta}}\left(\underline{v}_{\eta}\right)$ tends to $\underline{H}$ strongly in $L^{2}\left(\mathbb{R}^{3}\right)$, and we obtain that

$$
\frac{\pi}{4} \int_{\mathbb{R}}\left(\left|\underline{v}^{2}(x)\right|^{2}+\left|\underline{v}^{3}(x)\right|^{2}\right) \mathrm{d} x=\lim \inf \frac{1}{2} \int_{\mathbb{R}^{3}}\left|\underline{H_{d}^{\eta}}\left(\underline{v}_{\eta}\right)\right|^{2} \mathrm{~d} X .
$$

Therefore,

$$
\underline{\mathcal{E}}(\underline{v}) \leq \lim \inf \underline{\mathcal{E}}_{\eta}\left(\underline{v}_{\eta}\right) .
$$

(ii) Reconstruction. Let $\underline{u}_{0} \in \underline{H}^{1}\left(\Omega_{1} ; S^{2}\right)$. For $\eta>0$, we define $\underline{u}_{\eta}=\underline{u}_{0}$. Then,

$$
\underline{\mathcal{E}}_{\eta}\left(\underline{u_{\eta}}\right)=\frac{A^{2}}{2} \int_{\Omega_{1}}\left|\partial_{x} \underline{u}_{0}\right|^{2} \mathrm{~d} X+\frac{1}{2} \int_{\mathbb{R}^{3}}\left|H_{d}^{\eta}\left(\underline{u}_{0}\right)\right|^{2} \mathrm{~d} X-h_{a} \int_{\Omega_{1}} \underline{u}_{0} \cdot e_{1} \mathrm{~d} X .
$$

As in the previous step, we have:

$$
\left.\frac{1}{2} \int_{\mathbb{R}^{3}} \mid \underline{H_{d}^{\eta}} \underline{\underline{u_{0}}}\right)\left.\right|^{2} \mathrm{~d} X \longrightarrow \frac{\pi}{4} \int_{\Omega_{1}}\left(\left.\underline{\mid u_{0}^{2}}\right|^{2}+\left|\underline{u_{0}^{3}}\right|^{2}\right) \mathrm{d} X,
$$

that is

$$
\underline{\mathcal{E}}_{\eta}\left(\underline{u}_{\eta}\right) \longrightarrow \underline{\mathcal{E}}\left(\underline{u_{0}}\right) .
$$

This theorem gives the behavior of minimizers for the one dimensional limit case: the limit energy, for $u \in H^{1}(]-L, L\left[; S^{2}\right)$ is then $\mathcal{E}(u)=\underline{\mathcal{E}}\left(u \cdot \chi_{B^{2}(0,1)}\right)$ :

$$
\mathcal{E}(u)=\frac{\pi A^{2}}{2} \int_{]-L, L[}\left|\frac{\mathrm{d} u}{\mathrm{~d} x}\right|^{2} \mathrm{~d} x+\frac{\pi}{4} \int_{]-L, L[}\left(\left|u_{2}\right|^{2}+\left|u_{3}\right|^{2}\right) \mathrm{d} x-h_{a} \pi \int_{]-L, L[} v \cdot e_{1} \mathrm{~d} x,
$$

then, in order to find out the effective field, we write (see [11]):

$$
H_{e}=-\frac{\mathrm{d} \mathcal{E}}{\mathrm{d} u},
$$

it is to say

$$
H_{e}=\pi A^{2} \frac{\partial^{2} u}{\partial x^{2}}-\frac{\pi}{2}\left(u_{2} e_{2}+u_{3} e_{3}\right)+\pi h_{a} e_{1} .
$$

The boundary conditions for the minimizers are:

$$
\frac{\partial u}{\partial x}=0 \text { at } x=-L \text { and } x=L,
$$

since we minimize the energy without Dirichlet boundary conditions. 
Then, the limit dynamic system is obtain using the Landau Lifschitz combined with the new effective field $H_{e}$.

We perform the following rescaling in time $t^{\prime}=\frac{\pi}{2} t$ and in space $x^{\prime}=x / A \sqrt{2}$, we denote by $h_{a}^{\prime}=2 h_{a}$ the rescaled applied field. The resulting rescaled system is then given by (1.3) as we expected.

\section{EXISTENCE OF CANONICAL WALL PROFILES}

In this section we are interested in characterizing equilibrium states of the magnetization in a finite nano-wire when $h=0$, it is to say when there is no external magnetic field. The constant stable solutions are those equal to $-e_{1}$ or $e_{1}$, since the demagnetizing field behaves like a $\mathbb{R} e_{1}$ easy axes anisotropic field. We are interested in profiles describing one wall connecting a left hand side $-e_{1}$ domain to a right hand side $e_{1}$ domain. So, a priori, we look for solutions which can be written as:

$$
\left(\begin{array}{c}
\sin \theta_{0} \\
\cos \theta_{0} \cos \tau \\
\cos \theta_{0} \sin \tau
\end{array}\right)
$$

where $\theta_{0}$ is a non decreasing map from $]-\frac{L}{A \sqrt{2}}, \frac{L}{A \sqrt{2}}\left[\right.$ into $\mathbb{R}$ satisfying $\theta_{0}\left(-\frac{L}{A \sqrt{2}}\right)<0<\theta_{0}\left(\frac{L}{A \sqrt{2}}\right)$. If we write the energy of such a profile, we obtain:

$$
\mathcal{E}\left(\theta_{0}, \tau\right)=\frac{1}{2} \int_{-\frac{L}{A \sqrt{2}}}^{-\frac{L}{A \sqrt{2}}}\left(\left|\theta_{0}^{\prime}\right|^{2}+\left|\tau^{\prime}\right|^{2} \cos ^{2} \theta_{0}\right)+\frac{1}{2} \int_{-\frac{L}{A \sqrt{2}}}^{-\frac{L}{A \sqrt{2}}}\left|\cos ^{2} \theta_{0}\right|^{2}
$$

So in order to decrease the energy, we obtain minimizing walls assuming that $\tau^{\prime}=0$, that is for constant $\tau$. Without loss of generality (by rotational invariance), we assume that $\tau=0$. Therefore, we look for wall profiles which can be written as follows:

$$
\left.M_{0}(x)=\left(\begin{array}{c}
\sin \theta_{0} \\
\cos \theta_{0} \\
0
\end{array}\right), \forall x \in\right]-\frac{L}{A \sqrt{2}}, \frac{L}{A \sqrt{2}}[
$$

where $\theta_{0}$ is a non decreasing map from $]-\frac{L}{A \sqrt{2}}, \frac{L}{A \sqrt{2}}\left[\right.$ into $\mathbb{R}$ satisfying $\theta_{0}\left(-\frac{L}{A \sqrt{2}}\right)<0<\theta_{0}\left(\frac{L}{A \sqrt{2}}\right)$, and such that $M_{0}$ is a stationary solution to (1.3). In fact, we want $M_{0}$ to verify:

$$
\left.-M_{0} \wedge H_{e}-M_{0} \wedge\left(M_{0} \wedge H_{e}\right)=0, \forall x \in\right]-\frac{L}{A \sqrt{2}}, \frac{L}{A \sqrt{2}}[
$$

with

then, one has the following relation

$$
H_{e}=\frac{\partial^{2} M_{0}}{\partial x^{2}}-\cos \theta_{0} e_{2}
$$

$$
\left.-\theta_{0}^{\prime \prime}-\sin \theta_{0} \cos \theta_{0}=0, \forall x \in\right]-\frac{L}{A \sqrt{2}}, \frac{L}{A \sqrt{2}}[
$$

with on the boundary

$$
\theta_{0}^{\prime}\left(-\frac{L}{A \sqrt{2}}\right)=\theta_{0}^{\prime}\left(\frac{L}{A \sqrt{2}}\right)=0
$$


Setting $\gamma_{0}=\theta_{0}\left(\frac{L}{A \sqrt{2}}\right)\left(\gamma_{0}\right.$ is supposed to be non negative, that is we look for a non decreasing solution $\left.\theta_{0}\right)$, we have, integrating the equation (3.1) and using (3.2):

$$
\left(\theta_{0}^{\prime}\right)^{2}+\sin ^{2} \theta_{0}=\sin ^{2} \gamma_{0}
$$

The length of the nano-wire has to be such that the function $\theta_{0}$ goes from $-\gamma_{0}$ to $\gamma_{0}$. From formula (3.3), we deduce the length:

$$
\ell\left(\gamma_{0}\right)=\int_{-\gamma_{0}}^{\gamma_{0}} \frac{\mathrm{d} \theta}{\sqrt{\sin ^{2} \gamma_{0}-\sin ^{2} \theta}} .
$$

Using the length expression computed above, we prove now Theorem 1.2.

Proof. In order to ensure the existence of an equilibrium state as defined above, we must verify that there exists $\left.\gamma_{0} \in\right] 0, \pi / 2\left[\right.$ such that $\ell\left(\gamma_{0}\right)=2 \frac{L}{A \sqrt{2}}$ (the case $\gamma_{0}=\pi / 2$ corresponds to the constant solution $M_{2}=-e_{1}$, which does not describe a wall). First of all, one has

$$
\ell\left(\gamma_{0}\right)=2 \int_{0}^{1} \frac{\gamma_{0}}{\sqrt{\sin ^{2} \gamma_{0}-\sin ^{2} u \gamma_{0}}} \mathrm{~d} u,
$$

we then see that

$$
\lim _{\gamma_{0} \rightarrow \frac{\pi}{2}} \ell\left(\gamma_{0}\right)=+\infty .
$$

Let us prove that $\ell$ is a non decreasing map. We denote by

$$
F(\gamma, u)=\frac{\gamma}{\left(\sin ^{2} \gamma-\sin ^{2} u \gamma\right)^{\frac{1}{2}}}
$$

so that

For $u \in[0,1[$, we have

$$
\ell(\gamma)=2 \int_{0}^{1} F(\gamma, u) \mathrm{d} u
$$

$$
\frac{\partial F}{\partial \gamma}=\frac{1}{\left(\sin ^{2} \gamma-\sin ^{2} u \gamma\right)^{\frac{3}{2}}}(g(\gamma)-g(u \gamma))
$$

with $g(\tau)=\sin \tau(\sin \tau-\tau \cos \tau)$.

We remark that for $\tau \in[0, \pi / 2], \tau \mapsto \sin \tau$ is increasing, $\tau \mapsto-\tau \cos \tau$ is increasing, and $(\sin \tau-\tau \cos \tau)$ remains non negative, so $g$ is an increasing map on $[0, \pi / 2]$. Hence for $\gamma \in[0, \pi / 2]$ and for $u \in[0,1[, g(\gamma)-g(u \gamma)$ is non negative, therefore for $u \in\left[0,1[, \gamma \mapsto F(\gamma, u)\right.$ is non decreasing, that is $\ell$ is strictly increasing on $\left.] 0, \frac{\pi}{2}\right]$.

Then, the comparison could be done at the limit $\gamma_{0}=0$ :

$$
\begin{aligned}
\lim _{\gamma_{0} \rightarrow 0} \ell\left(\gamma_{0}\right) & =\lim _{\gamma_{0} \rightarrow 0} \int_{-1}^{1} \frac{\gamma_{0}}{\sin \gamma_{0}} \frac{\mathrm{d} u}{\sqrt{1-\frac{\sin ^{2}\left(u \gamma_{0}\right)}{\sin ^{2} \gamma_{0}}}} \\
& =\int_{-1}^{1} \frac{\mathrm{d} u}{\sqrt{1-u^{2}}} \\
& =\pi,
\end{aligned}
$$

then, we require that $2 \frac{L}{A \sqrt{2}}>\pi$.

Remark 3.1. In the case of nanowires, in order to describe a wall, the magnetization must point out the domain so we are in presence of a Bloch type wall (see [18]). 


\section{LiNeAR UNSTABILITY OF CANONICAL WALL PROFILES WITHOUT APPLIED FIELD}

Let us consider $L, A$ and $\theta_{0}>0$ given by Theorem 1.2. We denote by $M_{0}$ the canonical wall profile

$$
M_{0}(x)=\left(\begin{array}{c}
\sin \theta_{0}(x) \\
\cos \theta_{0}(x) \\
0
\end{array}\right)
$$

This profile is a static solution to (1.3) with $h_{a}=0$. We aim to calculate the linearized equation of (1.3) around $M_{0}$. In order to calculate this linearization taking into account that we are only interested in the perturbations of $M_{0}$ satisfying the constraint $|m|=1$, we describe the small perturbations of $M_{0}$ as follows:

$$
m(t, x)=r_{1} M_{1}+r_{2} M_{2}+\sqrt{1-r_{1}^{2}-r_{2}^{2}} M_{0},
$$

where the mobile frame $\left(M_{0}(x), M_{1}(x), M_{2}\right)$ is given by

$$
M_{0}(x)=\left(\begin{array}{c}
\sin \theta_{0}(x) \\
\cos \theta_{0}(x) \\
0
\end{array}\right), M_{1}(x)=\left(\begin{array}{c}
-\cos \theta_{0}(x) \\
\sin \theta_{0}(x) \\
0
\end{array}\right), M_{2}(x)=\left(\begin{array}{l}
0 \\
0 \\
1
\end{array}\right)
$$

for $x \in]-\frac{L}{A \sqrt{2}}, \frac{L}{A \sqrt{2}}[$.

The new unknown is now $r=\left(r_{1}, r_{2}\right)$ taking its values into $\mathbb{R}^{2}$ and the profile $M_{0}$ corresponds to $r=0$. We rewrite the Landau-Lifschitz system (1.3) in the unknown $r$ and we linearize this new equation around $r=0$. In the new unknowns, the effective field writes:

$$
\begin{aligned}
H_{e}= & g_{0} M_{0}+\left(2 \theta_{0}^{\prime} \partial_{x} r_{1}+r_{1} \theta_{0}^{\prime \prime}-\cos \theta_{0} \sin \theta_{0} r_{1}\right) M_{0} \\
& +\left(\partial_{x x} r_{1}+\cos ^{2} \gamma_{0} r_{1}\right) M_{1}+\partial_{x x} r_{2} M_{2}+\text { non linear terms }
\end{aligned}
$$

where

$$
g_{0}=\sin ^{2} \theta_{0}-\left(\theta_{0}^{\prime}\right)^{2} .
$$

After tedious calculations, we obtain the following linearized equation:

$$
\partial_{t} r=J\left(\begin{array}{c}
\left(\mathcal{L}-\cos ^{2} \gamma_{0}\right) r_{1} \\
\mathcal{L} r_{2}
\end{array}\right)
$$

with

- $J=\left(\begin{array}{cc}-1 & -1 \\ 1 & -1\end{array}\right)$

- $\gamma_{0}=\theta_{0}(L / A \sqrt{2})$;

- $\mathcal{L} r_{i}=-\partial_{x x} r_{i}+g_{0} r_{i}$, where $g_{0}$ is given by (4.2).

The linear unstability of the wall structure computed in the previous section is given by the study of the operator $\mathcal{L}$.

Proposition 4.1. $\mathcal{L}$ is a linear, positive operator. Its first eigenvalue, 0 , is associated to the eigenfunction $\cos \theta_{0}$ and its second eigenvalue, 1 , is associated to the eigenfunction $\sin \theta_{0}$.

Proof. We set: $f=\theta_{0}^{\prime} \tan \theta_{0}$, then

$$
\mathcal{L}=\ell^{*} \ell \text {, where } \ell=\partial_{x}+f,
$$

then, we can conclude that $\mathcal{L}$ is a positive operator and that $\cos \theta_{0}$ is in the kernel of $\mathcal{L}$. Thus 0 is the first eigenvalue of $\mathcal{L}$ and $\operatorname{Ker} \mathcal{L}=\mathbb{R} \cos \theta_{0}$. 
Furthermore, we have:

$$
\mathcal{L}\left(\sin \theta_{0}\right)=\sin \theta_{0},
$$

it is to say that 1 is an eigenvalue of $\mathcal{L}$ associated to the eigenfunction $\sin \theta_{0}$. In addition we remark that $\sin \theta_{0}$ vanishes once in the domain, so by Sturm-Liouville theorem, 1 is the second eigenvalue of $\mathcal{L}$.

We can now prove Theorem 1.3.

Proof. We first remark that by definition of the canonical profile, then $\gamma_{0}<\pi / 2$. Indeed $\gamma_{0}=\pi / 2$ should imply that $\theta_{0}$ is constant equal to $\pi / 2$ and does not satisfy the condition $\theta_{0}(-L / \sqrt{2} A)<0$. This case corresponds to the case $M_{0} \equiv e_{1}$, which is a stable (but constant) configuration.

From the previous proposition, since $-\cos ^{2} \gamma_{0}<0$, we conclude that $\mathcal{L}-\cos ^{2} \gamma_{0}$ has one strictly negative eigenvalue, then, zero is unstable for the linearized of (1.3) around $M_{0}$.

\section{Stabilization of Walls}

Now, we discuss the stabilization of $M_{0}$ by the command $H_{a}$ (the applied field). We recall that we introduced the following command:

$$
h_{a}=h_{a}(m)=\frac{-A}{\sqrt{2} L} \int_{-\frac{L}{A \sqrt{2}}}^{\frac{L}{A \sqrt{2}}} m_{1}(t, s) \mathrm{d} s .
$$

We want to prove that the profile $M_{0}$ is a stable stationary solution for the following system:

$$
\left\{\begin{array}{l}
\frac{\partial m}{\partial t}=-m \wedge H_{e}-m \wedge\left(m \wedge H_{e}\right) \\
\frac{\partial m}{\partial x}\left(-\frac{L}{A \sqrt{2}}\right)=\frac{\partial m}{\partial x}\left(\frac{L}{A \sqrt{2}}\right)=0 \\
H_{e}=\frac{\partial^{2} m}{\partial x^{2}}-m_{2} e_{2}-m_{3} e_{3}+h_{a}(m) e_{1} .
\end{array}\right.
$$

Proof. To start with, let us introduce the wall canonical profile $M_{0}=\left(\sin \theta_{0}, \cos \theta_{0}, 0\right)$ given in Theorem 1.2 . We recall that

$$
\begin{aligned}
& \theta_{0}^{\prime \prime}+\sin \theta_{0} \cos \theta_{0}=0 \\
& \theta^{\prime}\left(-\frac{L}{A \sqrt{2}}\right)=\theta^{\prime}\left(\frac{L}{A \sqrt{2}}\right)=0
\end{aligned}
$$

Furthermore, on $\left[-\frac{L}{A \sqrt{2}}, \frac{L}{A \sqrt{2}}\right]$,

$$
\cos ^{2} \theta_{0}-\left(\theta_{0}^{\prime}\right)^{2}=\cos ^{2} \gamma_{0}
$$

where $\gamma_{0}=\theta_{0}\left(\frac{L}{A \sqrt{2}}\right)$. In addition, we remark that $\theta_{0}$ is an odd map.

Since $h_{a}\left(M_{0}\right)=0$ (as $\theta_{0}$ is odd), we remark that $M_{0}$ is a stationary solution of (5.1). 


\subsection{First step: moving frame}

As in the previous section, in the spirit of [8], we will describe the problem in the moving frame

$$
\left(M_{0}(x), M_{1}(x), M_{2}\right)
$$

given by (4.1).

We write the solutions to (5.1) as:

$$
m(t, x)=M_{0}(x)+r_{1}(t, x) M_{1}(x)+r_{2}(t, x) M_{2}+\nu(r(t, x)) M_{0}(x)
$$

where $\nu(r)=\sqrt{1-r_{1}^{2}-r_{2}^{2}}-1$. In this moving frame, we get

$$
H_{e}=\left(g_{0}+a_{0}\right) M_{0}+\left(a_{1}+\tilde{a}_{1}\right) M_{1}+a_{2} M_{2}
$$

with

$$
\begin{aligned}
& g_{0}=\sin ^{2} \theta_{0}-\left(\theta_{0}^{\prime}\right)^{2} \\
& a_{0}=2 \theta_{0}^{\prime} \partial_{x} r_{1}+r_{1} \theta_{0}^{\prime \prime}-\cos \theta_{0} \sin \theta_{0} r_{1}+\partial_{x x} \nu-\nu\left(\theta_{0}^{\prime}\right)^{2}+\nu \sin ^{2} \theta_{0}+h(r) \sin \theta_{0}, \\
& a_{1}=\partial_{x x} r_{1}+\cos ^{2} \gamma_{0} r_{1}-S\left(r_{1}\right) \cos \theta_{0}, \\
& \tilde{a}_{1}=-2 \partial_{x} \nu \theta_{0}^{\prime}-\varphi(r) \cos \theta_{0}, \\
& a_{2}=\partial_{x x} r_{2},
\end{aligned}
$$

where

$$
S\left(r_{1}\right)=\frac{A}{\sqrt{2} L} \int_{-\frac{L}{A \sqrt{2}}}^{\frac{L}{A \sqrt{2}}} r_{1} \cos \theta_{0} \mathrm{~d} s, \quad \varphi(r)=-\frac{A}{\sqrt{2} L} \int_{-\frac{L}{A \sqrt{2}}}^{\frac{L}{A \sqrt{2}}} \nu(r) \sin \theta_{0} \mathrm{~d} s,
$$

and $h(r)=S\left(r_{1}\right)+\varphi(r)$.

Using these coordinates in the Landau-Lifschitz equation (3.2) and projecting on $M_{1}$ and $M_{2}$ yield:

$$
\partial_{t} r=\Lambda r+F\left(x, r, \partial_{x} r, \partial_{x x} r\right)
$$

where

$$
\Lambda r=\left(\begin{array}{cc}
-1 & -1 \\
1 & -1
\end{array}\right)\left(\begin{array}{c}
\tilde{\mathcal{L}}_{1}\left(r_{1}\right) \\
\mathcal{L}\left(r_{2}\right)
\end{array}\right)
$$

with

$$
\begin{aligned}
& \mathcal{L}=-\partial_{x x}+g_{0}, \\
& \tilde{\mathcal{L}}_{1}=\mathcal{L}-\cos ^{2} \gamma_{0}+\cos \theta_{0} S,
\end{aligned}
$$

and where the non linear part $F$ is given by

$$
F\left(x, r, \partial_{x} r, \partial_{x x} r\right)=F_{1}(r) \partial_{x x} r+F_{2}(r)\left(\partial_{x} r, \partial_{x} r\right)+F_{3}(x, r) \partial_{x} r+F_{4}(r)+F_{5}(r),
$$


with

- $F_{1} \in \mathcal{C}^{\infty}\left(\mathbb{R}^{2} ; \mathcal{M}_{2}(\mathbb{R})\right)$ :

$$
\begin{aligned}
F_{1}(r)= & \left(\begin{array}{cc}
\left(r_{2}\right)^{2}+(\nu(r))^{2}+2 \nu(r) & \nu(r)-r_{1} r_{2} \\
r_{1} r_{2}-\nu(r) & \left(r_{1}\right)^{2}+(\nu(r))^{2}+2 \nu(r)
\end{array}\right) \\
& +\left(\begin{array}{c}
-r_{1}-r_{2}-\nu(r) r_{1} \\
r_{1}-r_{2}-\nu(r) r_{2}
\end{array}\right) \nu^{\prime}(r) ;
\end{aligned}
$$

- $F_{2} \in \mathcal{C}^{\infty}\left(\mathbb{R}^{2} ; \mathcal{L}_{2}\left(\mathbb{R}^{2} ; \mathbb{R}^{2}\right)\right)$ :

$$
F_{2}(r)\left(\partial_{x} r, \partial_{x} r\right)=\left(\begin{array}{c}
-r_{1}-r_{2}-\nu(r) r_{1} \\
r_{1}-r_{2}-\nu(r) r_{2}
\end{array}\right) \nu^{\prime \prime}(r)\left(\partial_{x} r, \partial_{x} r\right)
$$

- $F_{3} \in \mathcal{C}^{\infty}\left(\left[-\frac{L}{A \sqrt{2}}, \frac{L}{A \sqrt{2}}\right] \times \mathbb{R}^{2} ; \mathcal{L}\left(\mathbb{R}^{2} ; \mathbb{R}^{2}\right)\right)$ :

$$
\begin{aligned}
F_{3}(x, r) \partial_{x} r= & 2 \theta_{0}^{\prime}(x)\left(\begin{array}{c}
-r_{1}-r_{2}-\nu(r) r_{1} \\
r_{1}-r_{2}-\nu(r) r_{2}
\end{array}\right) \partial_{x} r_{1} \\
& -2 \theta_{0}^{\prime}(x)\left(\begin{array}{c}
\left(r_{2}\right)^{2}+(\nu(r))^{2}+2 \nu(r)+1 \\
r_{1} r_{2}-\nu(r)-1
\end{array}\right) \nu^{\prime}(r)\left(\partial_{x} r\right) ;
\end{aligned}
$$

- $F_{4}(r) \in \mathcal{C}^{\infty}\left(\left[-\frac{L}{A \sqrt{2}}, \frac{L}{A \sqrt{2}}\right] \times \mathbb{R}^{2} ; \mathbb{R}^{2}\right)$ :

$$
\begin{aligned}
F_{4}(r)= & \left(\cos ^{2} \gamma_{0} r_{1}\right)\left(\begin{array}{c}
\left(r_{2}\right)^{2}+(\nu(r))^{2}+2 \nu(r) \\
r_{1} r_{2}-\nu(r)
\end{array}\right)-g_{0} \nu(r)\left(\begin{array}{c}
r_{1} \\
r_{2}
\end{array}\right) \\
& +\left(\left(\theta_{0}^{\prime \prime}-\cos \theta_{0} \sin \theta_{0}\right) r_{1}+g_{0} \nu(r)\right)\left(\begin{array}{c}
-r_{1}-r_{2}-\nu(r) r_{1} \\
r_{1}-r_{2}-\nu(r) r_{2}
\end{array}\right) ;
\end{aligned}
$$

- $F_{5}$ is given by

$$
\begin{aligned}
F_{5}(r)= & -h(r) \cos \theta_{0}\left(\begin{array}{c}
\left(r_{2}\right)^{2}+(\nu(r))^{2}+2 \nu(r) \\
r_{1} r_{2}-\nu(r)
\end{array}\right)-\varphi(r) \cos \theta_{0}\left(\begin{array}{c}
1 \\
-1
\end{array}\right) \\
& +h(r) \sin \theta_{0}\left(\begin{array}{c}
-r_{1}-r_{2}-\nu(r) r_{1} \\
r_{1}-r_{2}-\nu(r) r_{2}
\end{array}\right) .
\end{aligned}
$$

Remark 5.1. The command $h$ makes the linear part of (5.2) positive. Indeed, on one hand, we know that $\mathcal{L} \geq 0$ with $\operatorname{Ker} \mathcal{L}=\mathbb{R} \cos \theta_{0}$. On the other hand, $\tilde{\mathcal{L}}_{1}=\mathcal{L}+\cos \theta_{0} S-\cos ^{2} \gamma_{0}$. On $\mathbb{R} \cos \theta_{0}, \tilde{\mathcal{L}}_{1} \cos \theta_{0}=\alpha_{0} \cos \theta_{0}$ 
with

$$
\alpha_{0}=\frac{A}{\sqrt{2} L} \int_{-\frac{L}{A \sqrt{2}}}^{\frac{L}{A \sqrt{2}}} \cos ^{2} \theta_{0}(x) \mathrm{d} x-\cos ^{2} \gamma_{0}>0
$$

since for $x \in]-\frac{L}{A \sqrt{2}}, \frac{L}{A \sqrt{2}}\left[, \cos ^{2} \theta_{0}(x)>\cos ^{2} \gamma_{0}\right.$, and on $\left(\mathbb{R} \cos \theta_{0}\right)^{\perp}$,

$$
\left.\tilde{\mathcal{L}}_{1}\right|_{\left(\cos \theta_{0}\right)^{\perp}}=\mathcal{L}-\cos ^{2} \gamma_{0} \geq 1-\cos ^{2} \gamma_{0}
$$

since $\mathcal{L} \geq 1$ on $\left(\mathbb{R} \cos \theta_{0}\right)^{\perp}$ (see Prop. 4.1).

\subsection{Second step}

\subsubsection{New unknown}

The Landau Lifschitz equation (1.3) is invariant by rotation around the wire axis, so we can build a one parameter family of static solutions for (5.2).

For $\tau \in \mathbb{R}$ let us introduce the rotation around the $x$-axis given by

$$
\rho_{\tau}=\left(\begin{array}{ccc}
1 & 0 & 0 \\
0 & \cos \tau & -\sin \tau \\
0 & \sin \tau & \cos \tau
\end{array}\right)
$$

We denote $M_{\tau}(x)=\rho_{\tau} M_{0}(x)$, and $R_{\tau}$ its projection on the moving frame:

$$
R_{\tau}(x)=\left(\begin{array}{l}
M_{\tau}(x) \cdot M_{1}(x) \\
M_{\tau}(x) \cdot M_{2}(x)
\end{array}\right)=\left(\begin{array}{c}
\cos \theta_{0}(x) \sin \theta_{0}(x)(\cos \tau-1) \\
\cos \theta_{0}(x) \sin \tau
\end{array}\right) .
$$

Since $M_{\tau}$ is solution to (5.1), $R_{\tau}$ is a static solution of (5.2), that is

$$
\Lambda R_{\theta}+F\left(x, R_{\theta}, \partial_{x} R_{\theta}, \partial_{x x} R_{\theta}\right)=0 .
$$

Now in order to avoid the problems due to the zero eigenvalue of $\Lambda$, we describe $r$ in the new coordinates $(\tau, \sigma, W)$ defined by

$$
r(t, x)=R_{\tau(t)}(x)+\sigma(t)\left(\begin{array}{c}
\cos \theta_{0}(x) \\
0
\end{array}\right)+W(t, x),
$$

where $(\tau, \sigma) \in \mathcal{C}^{1}\left(\mathbb{R}^{+} ; \mathbb{R}^{2}\right)$ and $W \in \mathcal{C}^{1}\left(\mathbb{R}^{+} ; H^{2}\right)$ such that both coordinates of $W$ are in $\left(\mathbb{R} \cos \theta_{0}\right)^{\perp}$.

Indeed, as in [12], we can prove that for a given $r \in H^{2}\left(-\frac{L}{A \sqrt{2}}, \frac{L}{A \sqrt{2}}\right)$ in a neighbourhood of 0 , there exists a unique $(\tau, \sigma, W) \in \mathbb{R} \times \mathbb{R} \times \mathcal{W}$ such that

$$
\rho=R_{\theta}+\sigma\left(\begin{array}{c}
\cos \theta_{0} \\
0
\end{array}\right)+W
$$

where $\mathcal{W}$ is the set of the $W=\left(\begin{array}{l}W_{1} \\ W_{2}\end{array}\right) \in H^{2}$ satisfying the homogeneous Neumann Boundary condition $\frac{\partial W}{\partial x}\left(-\frac{L}{A \sqrt{2}}\right)=\frac{\partial W}{\partial x}\left(\frac{L}{A \sqrt{2}}\right)=0$ and the orthogonality condition:

$$
\int_{-\frac{L}{A \sqrt{2}}}^{\frac{L}{A \sqrt{2}}} W_{1}(x) \cos \theta_{0}(x) \mathrm{d} x=\int_{-\frac{L}{A \sqrt{2}}}^{\frac{L}{A \sqrt{2}}} W_{2}(x) \cos \theta_{0}(x) \mathrm{d} x=0 .
$$


Remark 5.2. On $\left(\mathbb{R} \cos \theta_{0}\right)^{\perp}$, the operators $\mathcal{L}$ and $\mathcal{L}-\cos ^{2} \theta_{0}$ are non negative, so we introduce the following norms on $\mathcal{W}$, respectively equivalent to the $H^{2}$ and the $H^{3}$ norms:

$$
\begin{aligned}
& \|W\|_{\mathcal{H}^{2}}=\left(\left\|\mathcal{L}^{\frac{1}{2}}\left(\mathcal{L}-\cos ^{2} \gamma_{0}\right)^{\frac{1}{2}} W_{1}\right\|_{L^{2}}^{2}+\left\|\mathcal{L} W_{2}\right\|_{L^{2}}^{2}\right)^{\frac{1}{2}} \\
& \|W\|_{\mathcal{H}^{3}}=\left(\left\|\mathcal{L}^{\frac{1}{2}}\left(\mathcal{L}-\cos ^{2} \gamma_{0}\right) W_{1}\right\|_{L^{2}}^{2}+\left\|\mathcal{L}^{\frac{3}{2}} W_{2}\right\|_{L^{2}}^{2}\right)^{\frac{1}{2}} .
\end{aligned}
$$

\subsubsection{Equation (5.2) with these unknowns}

Plugging the decomposition (5.5) in (5.2) and using (5.4) yield the following equivalent form for the LandauLifschitz equation in the coordinates $(\tau, \sigma, W)$, valid for small perturbations of $M_{0}$ :

$$
\frac{\mathrm{d} \tau}{\mathrm{d} t} R_{\tau}^{\prime}+\frac{\mathrm{d} \sigma}{\mathrm{d} t}\left(\begin{array}{c}
\cos \theta_{0} \\
0
\end{array}\right)+\partial_{t} W=\sigma\left(\begin{array}{c}
-\alpha_{0} \cos \theta_{0} \\
\alpha_{0} \cos \theta_{0}
\end{array}\right)+\Lambda W+G\left(x, \tau, \sigma, W, \partial_{x} W, \partial_{x x} W\right) .
$$

Let us describe the different terms in this equation:

Concerning the time derivatives, we have

$$
\partial_{t} r=\frac{\mathrm{d} \tau}{\mathrm{d} t} R_{\tau}^{\prime}(x)+\frac{\mathrm{d} \sigma}{\mathrm{d} t}\left(\begin{array}{c}
\cos \theta_{0} \\
0
\end{array}\right)+\partial_{t} W
$$

where

$$
R_{\tau}^{\prime}(x)=\left(\begin{array}{c}
-\sin \tau \cos \theta_{0} \sin \theta_{0} \\
\cos \tau \cos \theta_{0}
\end{array}\right)
$$

Concerning the linear part, we have

$$
\Lambda r=\Lambda R_{\tau}+\left(\begin{array}{c}
-\alpha_{0} \cos \theta_{0} \\
\alpha_{0} \cos \theta_{0}
\end{array}\right) \sigma+\Lambda(W)
$$

(see Rem. 4.1 for the definition of $\alpha_{0}$ ).

Concerning the non linear part, we have

$$
F\left(x, r, \partial_{x} r, \partial_{x x} r\right)=F\left(x, R_{\tau}, \partial_{x} R_{\tau}, \partial_{x x} R_{\tau}\right)+G\left(x, \tau, \sigma, W, \partial_{x} W, \partial_{x x} W\right)
$$

The last term $G$ is obtained from $F$ with the Taylor formula around $R_{\tau}$ :

$$
\begin{aligned}
G\left(x, \tau, \sigma, W, \partial_{x} W, \partial_{x x} W\right)= & F_{1}(r)\left(\partial_{x x} w\right)+\tilde{F}_{1}(r)(w)\left(\partial_{x x} R_{\tau}\right) \\
& +2 F_{2}(r)\left(\partial_{x} w, \partial_{x} R_{\tau}\right)+F_{2}(r)\left(\partial_{x} w, \partial_{x} w\right) \\
& +\tilde{F}_{2}(r)(w)\left(\partial_{x} R_{\tau}, \partial_{x} R_{\tau}\right) \\
& +F_{3}(x, r)\left(\partial_{x} w\right)+\tilde{F}_{3}(x, r)(w)\left(\partial_{x} R_{\tau}\right)+\tilde{F}_{4}(r)(w) \\
& +F_{5}(r),
\end{aligned}
$$

where

$$
\text { - } w=W+\left(\begin{array}{c}
\cos \theta_{0} \\
0
\end{array}\right) \sigma
$$


- for $i=1, \ldots, 4, \tilde{F}_{i}(r) \in \mathcal{L}\left(\mathbb{R}^{2} ; \mathbb{R}^{2}\right)$ is given by

$$
\tilde{F}_{i}(r)=\int_{0}^{1} F_{i}^{\prime}\left(R_{\tau}+s w\right) \mathrm{d} s .
$$

From straightforward calculations, we see that:

$$
\begin{aligned}
& F_{1}(r)=\mathcal{O}\left(|r|^{2}\right), \tilde{F}_{1}(r)=\mathcal{O}\left(|r|^{2}\right) \\
& F_{2}(r)=\mathcal{O}(|r|), \quad \tilde{F}_{2}(r)=\mathcal{O}(1) \\
& F_{3}(x, r)=\mathcal{O}(|r|), \quad \tilde{F}_{3}(x, r)=\mathcal{O}(1) \\
& \tilde{F}_{4}(r) \mathcal{O}(|r|) .
\end{aligned}
$$

- Concerning $F_{5}$, on one hand we remark that

$$
S\left(r_{1}\right)=S\left(W_{1}\right)+\sigma S\left(\cos \theta_{0}\right)
$$

(since $S\left(R_{\tau}\right)=0$ ).

On the other hand, $\varphi\left(R_{\tau}\right)=0$ and

$$
\varphi(r)=-\frac{\varepsilon}{2 L} \int_{-\frac{L}{A \sqrt{2}}}^{\frac{L}{A \sqrt{2}}} \tilde{\nu}(r)(w) \sin \theta_{0},
$$

where

$$
\tilde{\nu}(r)=\int_{0}^{1} \nu^{\prime}\left(R_{\tau}+s w\right) \mathrm{d} s=\mathcal{O}(|r|)
$$

Therefore with all these estimates, if $\|r\|_{H^{2}}$ is sufficiently small, we have

$$
\begin{gathered}
\|G\|_{L^{2}} \leq K\|r\|_{L^{\infty}}\left[|\sigma|+\|W\|_{\mathcal{H}^{2}}\right], \\
\left\|\partial_{x} G\right\|_{L^{2}} \leq K\|r\|_{L^{\infty}}\left[|\sigma|+\|W\|_{\mathcal{H}^{3}}\right]
\end{gathered}
$$

(see Rem. 5.2).

\subsubsection{Separation of the unknowns}

In order to separate the unknowns $\tau, \sigma$ and $W$, we first take the inner product of (5.6) with $\left(\begin{array}{c}\cos \theta_{0} \\ 0\end{array}\right)$ and with $\left(\begin{array}{c}0 \\ \cos \theta_{0}\end{array}\right)$. We remark that both $\partial_{t} W$ and $\Lambda W$ are orthogonal to these vectors, so that we obtain:

$$
\begin{aligned}
& \rho_{0} \sigma^{\prime}=-\alpha_{0} \rho_{0} \sigma+\bar{G}_{1}, \\
& g_{\tau} \tau^{\prime}=\alpha_{0} \rho_{0} \sigma+\bar{G}_{2},
\end{aligned}
$$


where

$$
\begin{aligned}
& \rho_{0}=\int_{-\frac{L}{A \sqrt{2}}}^{\frac{L}{A \sqrt{2}}} \cos ^{2} \theta_{0}, \\
& \bar{G}_{1}=\int_{-\frac{L}{A \sqrt{2}}}^{\frac{L}{A \sqrt{2}}} G\left(x, \tau, \sigma, W, \partial_{x} W, \partial_{x x} W\right) \cdot\left(\begin{array}{c}
\cos \theta_{0} \\
0
\end{array}\right) \mathrm{d} x, \\
& g_{\tau}=\int_{-\frac{L}{A \sqrt{2}}}^{\frac{L}{A \sqrt{2}}} R_{\tau}^{\prime}(x) \cdot\left(\begin{array}{c}
0 \\
\cos \theta_{0}
\end{array}\right) \mathrm{d} x, \\
& \bar{G}_{2}=\int_{-\frac{L}{A \sqrt{2}}}^{\frac{L}{A \sqrt{2}}} G\left(x, \tau, \sigma, W, \partial_{x} W, \partial_{x x} W\right) \cdot\left(\begin{array}{c}
0 \\
\cos \theta_{0}
\end{array}\right) \mathrm{d} x .
\end{aligned}
$$

By subtraction, we have:

$$
\partial_{t} W=\Lambda W+\widetilde{G}
$$

with

$$
\widetilde{G}=G-\frac{\bar{G}_{2}}{g_{\tau}} R_{\tau}^{\prime}-\frac{\bar{G}_{1}}{\rho_{0}}\left(\begin{array}{c}
\cos \theta_{0} \\
0
\end{array}\right)+\alpha_{0} \sigma\left[\left(\begin{array}{c}
0 \\
\cos \theta_{0}
\end{array}\right)-\frac{\rho_{0}}{g_{\tau}} R_{\tau}^{\prime}\right] .
$$

\subsubsection{Equivalent formulation}

We are then led to study the following equation

$$
\tau^{\prime}=\alpha_{0} \frac{\rho_{0}}{g_{\tau}} \sigma+\frac{1}{g_{\tau}} \bar{G}_{2},
$$

together with the system coupling:

$$
\sigma^{\prime}=-\alpha_{0} \sigma+\frac{1}{\rho_{0}} \bar{G}_{1}
$$

with

$$
\partial_{t} W=\left(\begin{array}{c}
-\left(\mathcal{L}-\cos ^{2} \gamma_{0}\right) W_{1}-\mathcal{L} W_{2} \\
\left(\mathcal{L}-\cos ^{2} \gamma_{0}\right) W_{1}-\mathcal{L} W_{2}
\end{array}\right)+\widetilde{G}
$$

From (5.7), with Remark 5.2 we have

$$
\left|\frac{1}{\rho_{0}} \bar{G}_{1}\right|(t) \leq K\|r\|_{L^{\infty}}\left[|\sigma(t)|+\|W(t)\|_{\mathcal{H}^{3}}\right] .
$$

In addition, since $g_{\tau}=\rho_{0}+\mathcal{O}\left(\tau^{2}\right)$, since $R_{\tau}^{\prime}=\left(\begin{array}{c}0 \\ \cos \theta_{0}\end{array}\right)+\mathcal{O}(\tau)$, we get:

$$
\|\tilde{G}\|_{H^{1}} \leq K\|r\|_{L^{\infty}}\left[|\sigma(t)|+\|W(t)\|_{\mathcal{H}^{3}}\right] .
$$

\subsection{Last step: Variational estimates}

Taking the inner product of (5.10) with $\left(\begin{array}{c}\mathcal{L}\left(\mathcal{L}-\cos ^{2} \gamma_{0}\right) W_{1} \\ \mathcal{L}^{2} W_{2}\end{array}\right)$, we obtain using (5.12) that

$$
\frac{\mathrm{d}}{\mathrm{d} t}\left(\|W\|_{\mathcal{H}^{2}}^{2}\right)+\|W\|_{\mathcal{H}^{3}}^{2} \leq K\|r\|_{L^{\infty}}\left[|\sigma(t)|+\|W(t)\|_{\mathcal{H}^{3}}\right] .
$$


Remark 5.3. In order to avoid the boundary terms when we integrate by part, we perform the previous estimates on the Galerkin approximation of (5.10) built on the eigenvectors of $\mathcal{L}$ with the homogeneous Neumann boundary condition.

Multiplying (5.9) by $\sigma,(5.11)$ yields:

$$
\frac{\mathrm{d}}{\mathrm{d} t} \sigma^{2}+\alpha_{0} \sigma^{2} \leq K\|r\|_{L^{\infty}}\left[|\sigma(t)|^{2}+\|W(t)\|_{\mathcal{H}^{3}}^{2}\right] .
$$

Summing up the previous estimates, we have:

$$
\frac{\mathrm{d}}{\mathrm{d} t}\left[|\sigma(t)|^{2}+\|W(t)\|_{\mathcal{H}^{2}}^{2}\right]+\alpha_{0}\left[|\sigma(t)|^{2}+\|W(t)\|_{\mathcal{H}^{3}}^{2}\right]\left(1-K\|r\|_{L^{\infty}}\right) \leq 0 .
$$

So there exists $\delta>0$ such that while $\|r\|_{L^{\infty}} \leq \frac{1}{2 K}$,

that is

$$
\frac{\mathrm{d}}{\mathrm{d} t}\left[|\sigma(t)|^{2}+\|W(t)\|_{\mathcal{H}^{2}}^{2}\right]+\delta\left[|\sigma(t)|^{2}+\|W(t)\|_{\mathcal{H}^{2}}^{2}\right] \leq 0
$$

$$
\left[|\sigma(t)|^{2}+\|W(t)\|_{\mathcal{H}^{2}}^{2}\right] \leq\left[\left|\sigma_{0}\right|^{2}+\left\|W_{0}\right\|_{\mathcal{H}^{2}}^{2}\right] \mathrm{e}^{-\delta t}
$$

Now, with equation (5.8), we have

$$
\left|\tau^{\prime}\right| \leq K|\sigma|+K\left[|\sigma(t)|+\|W(t)\|_{\mathcal{H}^{2}}\right]
$$

so while $\|r\|_{L^{\infty}} \leq \frac{1}{2 K}$,

$$
|\tau| \leq\left|\tau_{0}\right|+K\left[\left|\sigma_{0}\right|+\left\|W_{0}\right\|_{\mathcal{H}^{2}}\right] \mathrm{e}^{-\delta t / 2}
$$

Therefore, if $\tau_{0}, \sigma_{0}$ and $\left\|W_{0}\right\|_{H^{2}}$ are small enough, we remain in the domain $\left\{\|r\|_{L^{\infty}} \leq \frac{1}{2 K}\right\}$ and all the previous estimates remain valid for all times. This concludes the proof of Theorem 1.4.

\section{REFERENCES}

[1] F. Alouges, T. Rivière and S. Serfaty, Néel and cross-tie wall energies for planar micromagnetic configurations. ESAIM: COCV 8 (2002) 31-68.

[2] W.F. Brown, Micromagnetics. Interscience Publisher, John Willey and Sons, New York (1963).

[3] G. Carbou, Regularity for critical points of a nonlocal energy. Calc. Var. 5 (1997) 409-433.

[4] G. Carbou, Thin layers in micromagnetism. Math. Models Methods Appl. Sci. 11 (2001) 1529-1546.

[5] G. Carbou and P. Fabrie, Time average in micromagnetism. J. Differ. Equ. 147 (1998) 383-409.

[6] G. Carbou and P. Fabrie, Regular solutions for Landau-Lifschitz equation in a bounded domain. Differential Integral Equations 14 (2001) 213-229.

[7] G. Carbou and P. Fabrie, Regular solutions for Landau-Lifschitz equation in $\mathbb{R}^{3}$. Commun. Appl. Anal. 5 (2001) 17-30.

[8] G. Carbou and S. Labbé, Stability for static walls in ferromagnetic nanowires. Discrete Continous Dyn. Syst. Ser. B 6 (2006) 273-290.

[9] G. Carbou, S. Labbé and E. Trélat, Control of travelling walls in a ferromagnetic nanowire. Discrete Contin. Dyn. Syst. Ser. S 1 (2008) 51-59.

[10] A. DeSimone, R.V. Kohn, S. Müller and F. Otto, Magnetic microstructures - a paradigm of multiscale problems, in ICIAM 99 (Edinburgh), Oxford Univ. Press, Oxford (2000) 175-190.

[11] L. Halpern and S. Labbé, Modélisation et simulation du comportement des matériaux ferromagnétiques. Matapli 66 (2001) $70-86$. 
[12] T. Kapitula, Multidimensional stability of planar travelling waves. Trans. Amer. Math. Soc. 349 (1997) $257-269$.

[13] K. Kühn, Travelling waves with a singularity in magnetic nanowires. Commun. Partial Diff. Equ. 34 (2009) $722-764$.

[14] S. Labbé, Simulation numérique du comportement hyperfréquence des matériaux ferromagnétiques. Thèse de l'Université Paris 13, Paris (1998).

[15] S. Labbé and P.-Y. Bertin, Microwave polarisability of ferrite particles with non-uniform magnetization. J. Magn. Magn. Mater. 206 (1999) 93-105.

[16] T. Rivière and S. Serfaty, Compactness, kinetic formulation, and entropies for a problem related to micromagnetics. Commun. Partial Diff. Equ. 28 (2003) 249-269.

[17] D. Sanchez, Méthodes asymptotiques en ferromagnétisme. Thèse de l'Université Bordeaux 1, Bordeaux (2004).

[18] A. Thiaville, J.M. Garcia and J. Miltat, Domain wall dynamics in nanowires. J. Magn. Magn. Mater. 242-245 (2002) 10611063.

[19] A. Visintin, On Landau Lifschitz equation for ferromagnetism. Japan Journal of Applied Mathematics 1 (1985) 69-84.

[20] H. Wynled, Ferromagnetism, Encyclopedia of Physics XVIII/2. Springer-Verlag, Berlin (1966). 